\title{
EDITORIAL
}

\section{Potential for new medical countermeasures for radiation injury by targeting the Hedgehog signaling pathway}

Bone Marrow Transplantation (2014) 49, 321-323; doi:10.1038/ bmt.2013.203

lonizing radiation (IR) induces apoptosis in many cell types in a dose-dependent fashion. Of all cells of the body, the Sertoli cells of the testes are the most sensitive to IR-induced apoptosis and necrosis. Hematopoietic elements are also highly impacted by radiation effects. For example, the rate of reduction in the absolute lymphocyte count following exposure to IR correlates so well with cumulative radiation dose and dose rate that this decline is commonly used as a clinical surrogate marker for whole-body radiation dose. ${ }^{1,2}$ Hematopoietic progenitor cells are also reduced after exposure to IR with a Do (the dose at which survival is reduced to $e^{-1}$ or 0.37 of its previous value on the exponential portion of the survival curve) of $1.18 \pm 0.24 \mathrm{~Gy}$ for human circulating progenitors at a dose rate of $0.8 \mathrm{~Gy} / \mathrm{min}$, and $1.02 \pm 0.05 \mathrm{~Gy}$ for human BM-derived colony-forming unit (CFU)$\mathrm{GM}$ at a dose rate of $2.0 \mathrm{~Gy} / \mathrm{min}^{3,4}$ This pro-apoptotic effect leads to impaired myeloid differentiation and resultant neutropenia. Together with radiation-induced lymphopenia, impaired lymphokine gene expression and bacterial translocation across disrupted cutaneous and gastrointestinal mucosal barriers, neutropenia predisposes to fatal infections in irradiated humans. ${ }^{1,2,5}$

Hematopoietic cytokines have been used to mitigate the effect of radiation on hematopoietic progenitor/stem cell populations and attendant cytopenias. A review of the quality of evidence supporting this practice for management of the hematopoietic syndrome (defined as the development of one or more cytopenias in the setting of whole-body or significant partial-body acute radiation exposure) was undertaken by a panel of experts assembled in Geneva by the World Health Organization. ${ }^{6}$ Using the Grading of Recommendations Assessment Development and Evaluation (GRADE) system, data were abstracted from Englishlanguage articles identified in MEDLINE and PubMed. Although no randomized controlled trials of cytokines have been (or will likely be) conducted in irradiated humans, the quality of the evidence was found to be consistent with a beneficial effect on overall survival with a particular benefit in patients with exposure doses of $<5 \mathrm{~Gy}$, and the only likely critical organ failure is the hematopoietic system. On the basis of this analysis, a strong recommendation was made for the use of G-CSF or GM-CSF when the ANC is $<500 \times 10^{9}$ cells $/$ L. $^{6}$

In May 2013, this recommendation, together with newly published results showing efficacy of G-CSF in irradiated macaques, $^{7}$ was considered at a joint meeting of two subcommittees of the US Food and Drug Administration (FDA). The Medical Imaging Drugs Advisory Committee and the Oncologic Drugs Advisory Committee recommended that the FDA add radiation injury to the list of approved indications for this class of myeloid cytokines. Nevertheless, the Geneva consultancy recognized that new medical countermeasures are needed to prevent or enhance the recovery from radiationinduced myelosuppression, reduce infection rate and improve survival. $^{5}$
Hedgehog (hh) was first reported in 1980 as a gene that regulates the pattern of the embryonic cuticle of Drosophila. ${ }^{8}$ The sonic hh (Shh), Indian hh and desert hh genes were subsequently discovered in vertebrates. As recently reviewed by Jiang and Hui, humans were found to have a hh counterpart that is important for regulating tissue pattern formation and cell growth control. ${ }^{9} \mathrm{Hh}$ signaling has been implicated as a requisite for stem cell maintenance as well as for tissue repair and regeneration in adults. $^{10}$ The hh/GLI signaling pathway is involved in the regulation of stem cell activation and self-renewal, ${ }^{11}$ whereas errant activation of this pathway has been reported to contribute to the development of hematological malignancies (reviewed in Aberger et al. ${ }^{12}$ ) In addition to the Wnt and Notch signaling pathways, the Shh pathway has been implicated in the regulation of hematopoiesis, exerting an effect on the hematopoietic microenvironment through regulation of bone morphogenetic protein. ${ }^{13,14}$

In the February 2014 issue of BMT, Drouet et al. ${ }^{15}$ exploit the impact of Shh on the hematopoietic microenvironment to identify a new potential category of medical countermeasures for IRinduced myelosuppression. When irradiated nonhuman primates were given intraosseous porcine adipocyte-derived stem cells (ASCs) that underwent Shh gene transfer, the duration of absolute neutropenia, anemia and thrombocytopenia was significantly reduced, relative to that observed in irradiated nonhuman primates receiving mock-transfected ASCs.

Although the results are significant, the number of rhesus monkeys in test $(n=4)$, sham treated $(n=4$, two of which expired from radiation toxicity and were not evaluable for the hematological end points of this study) and control (administered no ASCs, $n=2,1$ of which expired) groups is small, raising the need for additional studies. Unfortunately, the nonhuman primate facility at the Institut de Recherche Biomedicale des Armees is slated for closure, leaving only a few research facilities wherein such studies can be performed in the future. Nevertheless, the intriguing possibility remains that chemicals or agents that alter or regulate sites in the hh/GLI signaling pathway of accessory cells may ultimately be identified as potential medical countermeasures for mitigation of radiation injury to the hematopoietic system.

Precisely how accessory cells in the microenvironment modulate the survival and growth of hematopoietic stem cells (HSCs) is unknown. The restrictive tissue microenvironment in which dormant HSCs reside is referred to as a niche. ${ }^{16}$ Two separate but not necessarily exclusive hypotheses suggest a role for osteoblasts located at the interface of marrow and bone or the endosteum (that is, the osteoblastic niche) and endothelial cells located at venous sinusoids where cells transit from the marrow to the circulation (that is, the vascular niche). Direct physical contact or soluble factors may mediate signaling from accessory cells to HSCs. ${ }^{17-22}$ In addition, signals may be trafficked both within the local microenvironment and throughout the circulation via plasma membrane-derived vesicles (more recently referred to as 'microvesicles' or 'exosomes') that are exfoliated in a directed, energy-dependent process from structurally distinct regions of the surface of accessory cells. ${ }^{23-27}$ We have shown that shed microvesicles carry cytokines, pro-apoptotic molecules (that 
are upregulated by exposure to IR) and MHC Ags on their surface, and that these regulatory molecules are enriched, compared with the activity expressed on parent membranes from which they arise. ${ }^{28-31}$ Recently, microvesicles have been shown to contain intravesicular microRNA whose biological significance is under investigation. ${ }^{32,33}$ As Shh has been found to upregulate angiogenic growth factors, ${ }^{34}$ it is tempting to speculate that its mechanism of action in hematopoiesis involves these intercellular signaling pathways within the vascular niche.

$\mathrm{Hh}$ is also involved in regulating the output from human pluripotent stem cells (iPS) to produce hematopoietic cells. Using human embryonic stem cell lines, Mcintyre et al. ${ }^{35}$ showed that transient GLI3-mediated inhibition of hh with chemical- and genetargeting-mediated inactivation of hh signaling resulted in initiation of the transition from embryonic to adult hematopoiesis. Although hh inhibition increased the total number of hematoendothelial progenitors, the potential of individual CFU-derived progeny from hematopoietic lineage progenitors was unchanged, consistent with an effect that is primarily directed at early-lineage fate decisions. Using induced human iPS, Amabile et al. ${ }^{36}$ generated teratomas in immunodeficient mice from which transplantable human hematopoietic cells develop, indicating for the first time that hematopoietic stem cells can be derived from human iPS cells. When iPS cells were co-transplanted with stromal cells, the number of CD34 + CD45 + cells was significantly increased in teratomas, suggesting that it is possible to modulate hematopoietic differentiation with accessory cells that are known to have a supportive role in hematopoiesis.

In theory, the promise of a personalized approach to medical care using patient-derived stem cells may be extended to individuals who are exposed to IR. ${ }^{37}$ For example, autologous iPS cells may be collected from somatic cells of individuals exposed to a high radiation dose, treated with a chemical that alters hh in favor of hematopoietic differentiation and returned to the exposed individual. Substantial hurdles must be overcome first, ranging from the consequences of random integration of viruses used to transduce somatic cells, including virus reactivation and stochastic activation of endogenous genes that predispose to human disease such as cancer, to malignant transformation of the transplanted cells, retention of genetic 'memory' from the somatic cell of origin and immune rejection. ${ }^{37,38}$ However, it may be feasible one day to manipulate hh in human iPS cells. Application of this technology to a large animal model may uncover yet additional methods to mitigate radiation injury to the hematopoietic system via hh with the ultimate goal of application to humans.

Translating promising molecular observations to clinical care requires assessment in large animals. To this end, governments should support nonhuman primate facilities that may be used to conduct mechanistic and efficacy studies of potential medical countermeasures in the setting of radiation exposure. Although the provocative findings reported by Drouet et al. require additional study, they raise the possibility that manipulation of hh in accessory cells of the marrow microenvironment, stem cells and/or hematopoietic lineage progenitor cells may one day yield a new class of medical countermeasures for the prevention and/or treatment of the hematopoietic syndrome resulting from radiation exposure.

\section{CONFLICT OF INTEREST}

The author declares no conflict of interest.

N Dainiak

Clinical Professor of Medicine, Yale University School of Medicine, New Haven, CT, USA

E-mail: NickDainMD@aol.com

\section{REFERENCES}

1 Dainiak N, Waselenko JK, Armitage JO, MacVittie TJ, Farese AM. The hematologist and radiation casualties. Hematol Am Soc Hematol Educ Program 2003; 473-496.

2 Waselenko JK, MacVittie TJ, Blakely WF, Pesik N, Wiley AL, Dickerson WE et al. Strategic National Stockpile Radiation Working Group. Medical management of acute radiation syndrome: recommendations of the Strategic National Stockpile Radiation Working Group. Ann Intern Med 2004; 140: 1037-1051.

3 FitzGerald TJ, McKenna M, Rothstein L, Daugherty C, Kase K, Greenberger JS. Radiosensitivity of human bone marrow granulocyte-macrophage progenitor cells and stromal colony-forming cells: effect of dose rate. Radiat Res 1986; 107 205-215.

4 Oriya A, Takahashi K, Inanami O, Miura T, Abe Y, Kuwabara M et al. Individual differences in the radiosensitivity of hematopoietic progenitor cells detected in steady-state human peripheral blood. J Radiat Res 2008; 49: 113-121.

5 Dainiak N, Gent RN, Carr Z, Schneider R, Bader J, Buglova E et al. Literature review and global consensus on management of acute radiation syndrome affecting nonhematopoietic organ systems. Disaster Med Public Health Prep 2011; 5: $183-201$

6 Dainiak N, Gent RN, Carr Z, Schneider R, Bader J, Buglov E et al. First global consensus for evidence-based management of the hematopoietic syndrome resulting from exposure to ionizing radiation. Disaster Med Public Health Prep 2011; 5: 202-212.

7 Farese AM, Cohen MV, Katz BP, Smith CP, Gibbs A, Cohen DM et al. Filgrastim improves survival in lethally irradiated nonhuman primates. Radiat Res 2013; 179 . 89-100.

8 Nusslein-Volhard C, Wieschaus E. Mutations affecting segment number and polarity in Drosophila. Nature 1980; 287: 795-801.

9 Jiang J, Hui C-c. Hedgehog signaling in development and cancer. Dev Cell 2008; 15: $801-812$.

10 Heretsch P, Tzagkaroulaki L, Giannis A. Modulators of the hedgehog signaling pathway. Bioorg Med Chem 2010; 18: 6613-6624.

11 Beachy PA, Karhadkar SS, Berman DM. Tissue repair and stem cell renewal in carcinogenesis. Nature 2004; 432: 324-331.

12 Aberger F, Kern D, Greil R, Hartmann TN. Canonical and non-canonical hedgehog/ GU signaling in hematological malignancies. Vitam Horm 2012; 88: 25-54.

13 Bhardwaj G, Murdoch B, Wu D, Baker DP, Williams KP, Chadwick K et al. Sonic hedgehog induces the proliferation of primitive human hematopoietic cells via BMP regulation. Nat Immunol 2001; 2: 172-180.

14 Blank U, Karissdon G, Karisson S. Signaling pathways governing stem-cell fate. Blood 2008; 111: 492-503.

15 Drouet M, Garrigou P, Peinnequin A, Hérodin F. Short-term sonic-hedgehog gene therapy to mitigate myelosuppression in highly irradiated monkeys: hype or reality? Bone Marrow Transplant 2014; 49: 304-309.

16 Morrison SJ, Spradling AC. Stem cells and niches: mechanisms that promote stem cell maintenance throughout life. Cell 2008; 132: 598-611.

17 Wagner W, Safrich R, Wirkner U, Eckstein V, Blake J, Ansorge A et al. Hematopoietic progenitor cells and cellular microenvironment: behavioral and molecular changes upon interaction. Stem Cells 2005; 23: 1180-1191.

18 Ellis SL, Grassinger J, Jones A. The relationship between bone, hemopoietic stem cells, and vasculature. Blood 2011; 118: 1516-1524.

19 Iwasaki H, Arai F, Kubota Y, Dahl M, Suda T. Endothelial protein C receptorexpressing hematopoietic stem cells reside in the perisinusoidal niche in fetal liver. Blood 2010; 116: 544-553.

20 Zhang CC, Kaba M, Ge G, Xie K, Tong W, Hug C et al. Angiopoietin-like proteins stimulate ex vivo expansion of hematopoietic stem cells. Nat Med 2006; 12 240-245.

21 Chou S, Lodish HF. Fetal liver hepatic progenitors are supportive stromal cells for hematopoietic stem cells. Proc Natl Acad Sci USA 2010; 107: 7799-7804.

22 Chou S, Flygare J, Lodish HF. Fetal hepatic progenitors support long-term expansion of hematopoietic stem cells. Exp Hematol 2013; 41: 479-490.

23 Dainiak N, Cohen CM. Surface membrane vesicles from mononuclear cells stimulate erythroid stem cells to proliferate in culture. Blood 1982; 60: 583-594.

24 Dainiak N. Surface membrane-associated regulation of cell assembly, differentiation and growth. Blood 1991; 78: 264-276.

25 Armstrong MJ, Storch J, Dainiak N. Structurally distinct plasma membrane regions give rise to extracellular membrane vesicles in normal and transformed lymphocytes. Biochim Biophys Acta 1988; 946: 106-112.

26 Dainiak N, Sorba S. Intracellular regulation of the production of human erythroiddirected lymphokines. J Clin Invest 1991; 87: 213-220.

27 Albanese J, Dainiak N. Modulation of intercellular communication mediated at the cell surface and on extracellular, plasma membrane-derived vesicles by ionizing radiation. Exp Hematol 2003; 31: 455-464.

28 Tuck D, Cerretti DP, Hand A, Guha A, Sorba S, Dainiak N. Human M-CSF is expressed and shed from the cell surface. Blood 1994; 84: 2182-2188. 
29 Albanese J, Meterissian S, Kontogiannea M, Dubreuil C, Hand A, Sorba S et al. Biologically active Fas antigen and its cognate ligand are expressed on plasma membrane-derived extracellular vesicles. Blood 1998; 91: 3862-3874.

30 Guha A, Dainiak N, Freedman M, Goldschneider I, Cone RE. Regulatory effect of interferon-gamma and phorbol esters on the surface expression and biosynthesis of MHC class-I antigens by human leukemia cells. Cell Immunol 1993; 151: 404-413.

31 Albanese J, Dainiak N. lonizing radiation alters Fas ligand at the surface and on exfoliated plasma membrane-derived vesicles: implications for apoptosis and intercellular signaling in vitro. Radiat Res 2000; 153: 49-61.

32 Woloschak G. Use of archival material to study miRNAs. ConRad 2013, Global Conference on Radiation Topics)Munich, Germany 2013.

33 Szabo G. Circulating exosomes in liver disease, Exosomes and Microvesicles as Biomarkers and Diagnostics, Philadelphia, PA, USA, 2013.
34 Pola R, Ling LE, Silver M, Corbley MJ, Kearney M, Blake Pepinsky R et al. The morphogen sonic hedgehog is an indirect angiogenic agent upregulating two families of angiogenic growth factors. Nat Med 2001; 7: 706-711.

35 Mcintyre BAS, Ramos-Mejia V, Rampali S, Mechael R, Lee J-H, Alev C et al. Gli3-mediated hedgehog inhibition in human pluripotent stem cells initiates and augments developmental programming of adult hematopoiesis. Blood 2013; 121: 1543-1552.

36 Amabile G, Welner RS, Nombela-Arrieta C, D'Alise AM, Di Ruscio A, Ebralidze AK et al. In vivo generation of transplantable human hematopoietic cells from induced pluripotent stem cells. Blood 2013; 121: 1255-1264.

37 Ess KC. Patient heal thyself: modeling and treating neurological disorders using patient-derived stem cells. Exp Biol Med 2013; 238: 308-314.

38 Ferreira LMR, Mostajo-Radji MA. How induced pluripotent stem cells are redefining personalized medicine. Gene 2013; 520: 1-6. 